\title{
Reply to the letter by I. Tasci regarding 'Clinical usefulness of AGEs as a biomarker for the attenuation of NASH'
}

\author{
Hideyuki Hyogo
}

Received: 25 February 2010/ Accepted: 26 February 2010/Published online: 24 March 2010

(C) Springer 2010

We appreciate the letter from Professor Ilker Tasci, M.D., showing his interest in our article and making some comments. He pointed that about one-third of the study population had overt diabetes mellitus. Because the base levels of $\mathrm{HbA} 1 \mathrm{c}$ were less than 6.5 in the studied population at entry to the study, 75-g oral glucose tolerance tests were undertaken to examine the prevalence of postprandial hyperglycemia and diabetes mellitus. Accordingly, antidiabetic medications were not administered; only ordinary diet counseling was provided. As a result, none of the patients experienced deteriorating conditions during the study, as shown in the tables in the article. As to the relatively high prevalence of hypertensive individuals, $63 \%$ of patients had already been treated properly with calcium antagonists (amlodipine), and this did not change during the study period. The rest of the hypertensive individuals were not treated because their blood pressure levels were slightly above the normal limit. For these reasons, we could not present adjusted information. In this study, because the changes in AGEs levels were not associated with impaired glucose tolerance, we would like to conclude that not only the diabetic condition, but also the oxidative situation affects the AGEs levels, and AGEs might become a candidate biomarker for NASH without overt diabetes mellitus (HbA1c less than 6.5). As Dr. Tasci pointed out, there are reports about the influence of AGEs level medications for diabetes mellitus and hypertension, including our reports. Finally, Dr. Tasci commented that "atorvastatin should be taken as a safe agent for dyslipidemia management in people with NASH, but more is needed to indicate it as an agent to 'treat' NASH with dyslipidemia for ALT normalization and histological recovery." This comment is important, but, because of the small numbers of samples and less recovery of fibrosis, further analyses and randomized placebo-controlled trials are needed. At least we have shown that atorvastatin is a safe agent and has some beneficial effects on histological recovery when managing dyslipidemia patients with NASH.

This author's reply refers to the letter to the editor at doi:10.1007/s00535-010-0229-1.

\footnotetext{
H. Hyogo $(\bowtie)$

Department of Medicine and Molecular Science, Graduate School of Biomedical Sciences, Hiroshima University, 1-2-3 Kasumi, Minamiku, Hiroshima 734-8551, Japan e-mail: hidehyogo@hiroshima-u.ac.jp
} 